\title{
Neue Leistungsklassen
}

\section{Liebe Leserin, lieber Leser,}

\section{„ZF ProAI“ und „Nvidia Drive PX 2 AI“} sind die neuen Stars des Friedrichshafener Antriebs- und Fahrwerktechnikspezialisten, präsentiert auf der Consumer Elektronics Show (CES) in Las Vegas Anfang des Jahres. Dabei handelt es sich nicht etwa um eine neue Getriebegeneration, die mit optimierten Antriebsleistungen beworben wird, sondern um integrierte Rechnerleistung einer neuen Dimension. Supercomputer und künstliche Intelligenz - Artificial Intelligence (AI) - stehen im Fokus des Interesses der Automobilisten sowie der ZF-Gruppe, die sich mit dem Kauf von TRW weiteres Know-how in der aktiven und passiven Sicherheitstechnik zugekauft hat. Mit leistungsfähigeren Rechnern und AI lassen sich die Entwicklungen des autonomen Fahrens drastisch beschleunigen.

Der Auftritt von ZF in Las Vegas steht symbolisch für den Wandel in der Automobil- und Mobilitätsbranche. Über diesen Wandel ist zwar schon viel geschrieben und geredet worden, dennoch bleiben viele Fragen unbeantwortet - und die Diskussionen in einem andauernden Findungsund Veränderungsprozess sehr spannend.

Es wird beispielsweise neue Rollen geben und damit Gewinner, aber auch Verlierer. Ein ehemaliger OEM-Elektronikchef bemerkte am Rande eines Interviews mit ATZelektronik schon vor Jahren, dass seine Abteilung beispielsweise nur als Dienstleister der Motorenentwickler verstanden wird. Eigentlich müsse es umgekehrt sein. Das Image der Berufsstände der Software-, IT- und ElektronikSpezialisten ist nach Meinung vieler Branchenexperten unterbewertet. Mit der neuen Position des Chief Digital Officers (CDO) kommt jetzt Bewegung in die Organigramme. Die CDOs sollen aber nicht die Umsetzer sein, sondern die Gestalter, mahnen Experten.

Die ZF-Pressekonferenz in Las Vegas lädt zu einer weiteren Betrachtung ein.
Welchen Stellenwert haben EnablerTechniken wie leistungsfähige Halbleiter? Eigentlich einen hohen. Doch lässt sich das Spezialthema nicht besonders effektvoll verkaufen. ZF zählt zu einer der wenigen Firmen, die das in der MarketingKommunikation mal anders angepackt haben. Chiphersteller Nvidia unterstützt dies ebenfalls werbewirksam mit schauspielerischem Talent vor 6000 Zuschauern in einer der CES-Eröffnungsreden.

Die CES zählt mittlerweile zu den entscheidenden Leistungsshows für die Automobilindustrie. Und besonders deutsche Besucher fragen sich, ob es in der Heimat eine vergleichsweise wichtige Veranstaltung für Automobilisten mit diesem Zuschnitt gibt. Nein, leider nicht. Es ist zwar zu spät, der etablierten CES Konkurrenz zu machen. Dennoch könnte man darüber nachdenken, wie zukunftsweisende Spezialthemen nicht nur unter Spezialisten diskutiert, sondern einem breiteren Publikum und Entscheidern zugänglicher gemacht werden können. Kryptische Bezeichnungen für Prozessoren werden dann vielleicht klangvollere Namen erhalten. Ein Kürzel wird sich dennoch einprägen: AI für Artificial Intelligence.

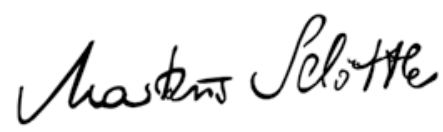

Markus Schöttle

Stellvertretender Chefredakteur

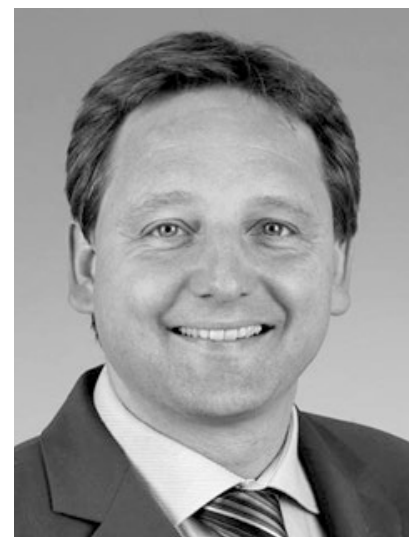

ESG. ENGINEERING AUF DER ÜBERHOLSPUR.

Als Ihr Partner für komplexe Elektronik- und IT-Systeme arbeiten wir mit Vollgas an der automobilen Zukunft. Mit Technologiekompetenz und besonderer Kundennähe liefern wir maßgeschneiderte und smarte Systemlösungen für die Mobilität von Morgen. Die ESG ist dabei Engineering-Partner, ProzessKnow-how-Träger, Technologieberater und IT-Experte in einem.

DEDICATED TO SOLUTIONS. WWW.ESG.DE

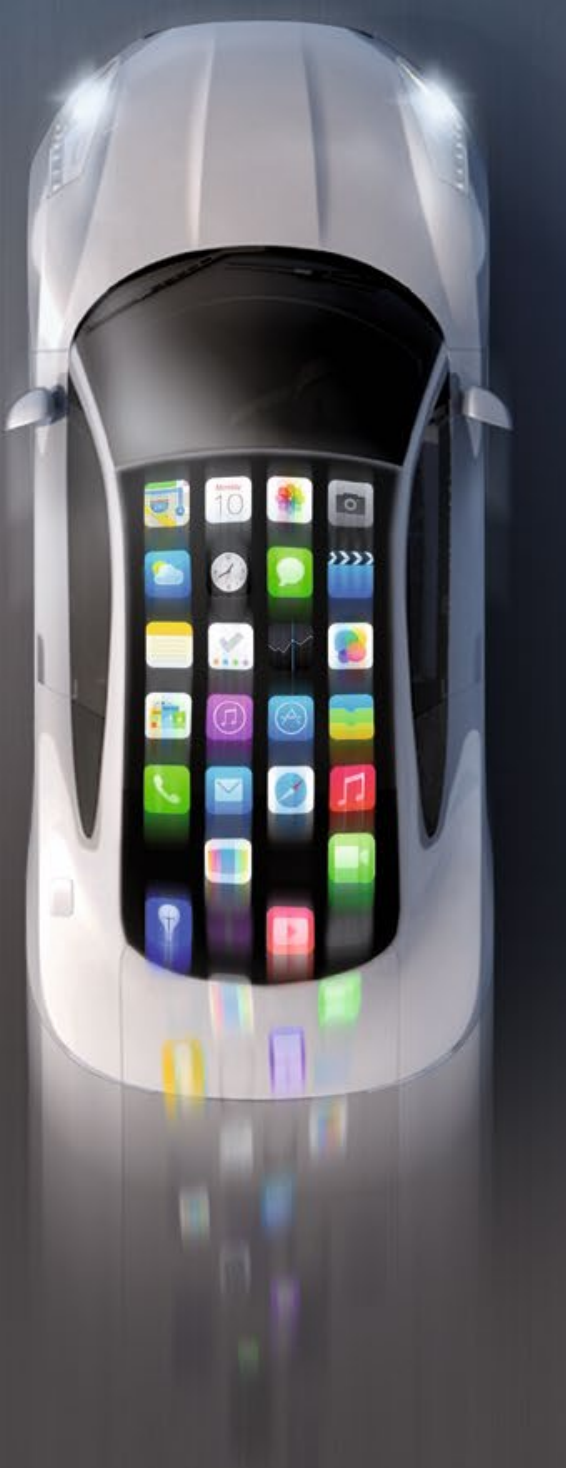

